\section{Zika Virus: A Zig Zag Path Ahead for India}

\author{
Neha Gautam ${ }^{1}$, Kapil Goyal ${ }^{2}$ and Mini P Singh ${ }^{1 *}$ \\ ${ }^{1}$ Department of Virology, Postgraduate Institute of Medical Education \& \\ Research, Chandigarh, India \\ ${ }^{2}$ Department of Medical Parasitology, Postgraduate Institute of Medical \\ Education and Research, Chandigarh, India
}

\begin{abstract}
Zika virus an arboviral infection, is an important public health problem. Recently, World Health Organization has declared it as 'Public Health Emergency of International Concern'. Zika virus belongs to Flaviviridae family, transmitted by Aedes aegypti mosquito. Though, it was discovered way back in 1947 from Zika forest in Uganda, but it gained importance in the year 2015 when a possible link with microcephaly was seen in the Brazil outbreak. In India, arboviral infections transmitted by Aedes mosquitoes are highly prevalent, hence India may be a fertile ground for the transmission of Zika if introduced in the country due to globalization. At present no vaccine or treatment is available. Thus, research is required to study the pathogenesis of microcephaly in order to develop the therapeutic targets. Efforts should be made to develop a point of care test along with a commitment to contain vector population by adopting vector control measures as it is the best preventive measure available at moment.
\end{abstract}

\section{Keywords: Zika}

Zika Virus (ZIKV) is an arboviral infection transmitted by the bite of infected Aedes mosquitoes, commonly Aedes aegypti and also by Aedes albopictus [1]. The other mosquito species occasionally implicated are Aedes henselli and Aedes polynesiensis. Recently, World Health Organisation (WHO) has declared an alert related to the pandemic potential of Zika virus [2]. The situation may deteriorate owing to this year's El Nino weather patterns which are expected to increase mosquito populations in many areas [3]. The WHO has already predicted that four million people could be infected with Zika in the Americas in 2016 [4]. On $1^{\text {st }}$ February 2016, the WHO director Margaret Chan declared Zika as "Public Health Emergency of International Concern" [2]. Thus, seriousness of Zika infection can be estimated from the fact that WHO has kept Zika virus under the same category as that of Ebola virus infection. The epicentre of the disease is Brazil and this caused scare and anxiety among the athletes and fans participating in the South America's first Olympics being held at Rio de Janeiro in August 2016 [5]. However so far the WHO has not reported any laboratory

*Corresponding author: Mini P Singh, Department of Virology, Postgraduate Institute of Medical Education \& Research, Chandigarh, India, Tel: +91 0172 2755170; Fax: +91 01722744401; E-mail: minipsingh@gmail.com

Citation: Gautam N, Goyal K, Singh MP (2016) Zika Virus: A Zig Zag Path Ahead for India. J Infect Non Infect Dis 2: 017.

Received: March 15, 2016; Accepted: October 12, 2016; Published: October 26, 2016 confirmed cases of Zika virus associated with the Olympics.

The Zika virus was discovered in the Zika forest of Uganda in the year 1947 from a rhesus monkey; this was followed by the detection of human cases in Uganda in 1952 [6,7]. Since its discovery, Zika virus had been of little importance as only 14 cases had been documented prior to the Yap island outbreak [6] and also because its disease severity was quite mild as compared to dengue and chikungunya which were prevalent in those areas. After years of silence, the first outbreak was reported in the Yap island (Federated States of Micronesia in 2007) [8], which was followed by larger epidemic in French Polynesia in South Pacific in 2013 and 2014 [8,9]. This was followed by smaller outbreaks in many islands of Africa, South East Asia and Pacific islands [8]. In May 2015, the Pan American Health Organization (PAHO) issued an alert when ZIKV emerged for the first time in Brazil. The emergence of ZIKV was associated with reports of neurological complications like Guillian Barre Syndrome in French Polynesia and microcephaly in neonates in Brazil [10-12]. It has a widespread pandemic potential as many cases have been documented from different geographical regions. Currently, outbreaks are occurring in many countries of Americas, Oceania/Pacific Islands (American Samoa, Samoa), and Africa (Cape Verde) [13]. The clinical severity varies from mild infection to severe infection presenting as microcephaly in newborns when the disease affects pregnant women. Since October 2015, more than 4,000 cases of microcephaly have been reported in Brazil alone [14]. Currently, no antiviral or vaccine is available for effective management of cases and susceptible population.

India is a fertile ground for Zika virus as the mosquitoes including Aedes aeygpti are present in abundance as has been documented by a high number of dengue and chikungunya cases being reported in recent years [15-18]. However, Zika is relatively new, and most of the population is not immune, hence the infection may spread at an alarming rate leading to high disease severity. The National Institute of Virology (NIV) study performed way back in 1950s documented that only 33 of the 196 people tested had immunity against Zika virus. Thus, large segment of population seems to be susceptible to Zika infection [19].

Dengue Virus (DENV) and ZIKV, both belong to Flaviviridae family and published reports have shown a high degree of antigenic similarity between the two viruses [20,21]. Studies using a panel of Human Monoclonal Antibodies (HMAbs) to DENV have reported that antibodies to DENV envelope protein also cross reacted with ZIKV but do not neutralize ZIKV thereby greatly enhancing ZIKV infection by antibody dependent enhancement in vitro [21,22].

Though, not even single case has been reported from India but as a precautionary measure, Government of India has issued general preventive guidelines in public interest [23]. Moreover, India also has a large pool of pregnant women that are susceptible to Zika infection, which can subsequently lead to the development of microcephaly among new-borns as observed in Brazil. As per Open Government Data (OGD) Platform India, more than 20 million pregnant women were enrolled for complete three antenatal check-ups during the year 
2010-2011 in India [24]. Thus, the total number of pregnant women in India are even more than the figure quoted above, and once Zika virus enters the Indian subcontinent, it may cause panic among these pregnant women [17]. An important concern here will be that once the Zika is introduced into the Indian subcontinent, further spread may also occurs by non-vector borne transmission. The semen has been shown to harbour high amount of Zika virus RNA and both male to female and female to male transmission has been reported. Apart from this, blood borne transmission has also been reported in endemic areas $[25,26]$. Screening of blood would be necessary to prevent transfusion transmitted infection. Though no FDA licenced tests are available for ZIKV, testing is available through two Investigational New Drug (IND) applications for blood collected in Puerto Rico and Mainland United States [26].

The past few years have been the era of viruses, and many new viruses have emerged and re-emerged including Severe Acute Respiratory Syndrome Coronavirus (SARS-CoV), Middle East Respiratory Syndrome Coronavirus (MERS-CoV), chikungunya, Avian influenza, Pandemic influenza (H1N1), and recently the Ebola virus [27]. It is pertinent to note that all these are RNA viruses, which are more prone to genetic mutations as compared to DNA viruses. Most of these diseases are zoonotic in origin due to increased human and animal interface [27].

Moreover, due to globalization, there has been a tremendous increase in the international travel. Thus, chances of spreading the infection from one geographical area to a distant location are very high [28]. India has been put on high alert after 13 Indians residing in Singapore were confirmed to have Zika virus infection. Hence, screening is necessary at international airport terminals as was performed during the Ebola outbreak. Also aircraft disinfection as per WHO recommendations would be pertinent in this regards as the transport of infected mosquitoes to the non-endemic area may lead to the spread of infection as demonstrated by numerous cases of "airport malaria" from Europe, North America and elsewhere [29,30].

It is still not clear if multiple arboviruses can be transmitted by a single mosquito. Since many arboviruses are endemic at a given geographical location, the theoretical possibility of the same cannot be ruled out. Though, dual infection with dengue and chikungunya has been documented in the past but it is not clear whether it is caused by a single mosquito infected with both viruses or by two different mosquitoes [31,32]. Moreover, laboratory experiments did not detect dual infection of A. aegypti with dengue and chikungunya from the same pool or from individual mosquitoes. It is known that both viruses have different incubation period in the mosquitoes and the one with shorter incubation period is at advantage of being transmitted with higher frequency [33]. However, dual infection with dengue and Zika virus have been reported in 2 patients in the New Caledonia outbreak in the year 2014 [34].

Therefore, the path ahead is not easy, and the scientific community is struggling to find the answers to the following:

1) What is the pathogenesis of the microcephaly induced by the Zika virus? Though a definitive link has been seen in some cases, the question to be answered remains is whether all these cases are caused by Zika or is it just a coincidence? An effective animal model may assist in understanding the pathogenesis of the virus. Till date there are three papers which mark an important turning point in Zika virus pathogenesis and enable in vivo testing and evaluation of candidate vaccines and therapeutics. Female immuno-deficient mice lacking the IFNa/B receptor (A129) and congenic control mice (129SV/EV) for ZIKV infection have been used for studying pathogenesis of Zika virus. While Dowall et al., [35] utilized type-1 interferon receptor deficient mice (A129) and showed that these mice are susceptible to Zika. Lazear et al., [36] showed that knockout mice lacking components of the antiviral response developed neurological disease and succumbed to ZIKV infection. Rossi et al., [37] demonstrated that the A129 mice model can also recapitulate the disease with exaggerated signs.

2) What is the pathogenesis of Guillain Barre (GBS) Syndrome? In the French Polynesia outbreak and Brazil, these was an increased number of cases of GBS observed in the Zika virus season and also neutralising antibodies to Zika virus were identified. The AXL receptor has been identified as a candidate Zika virus entry receptor in neural stem cells [38]. However, the exact pathogenesis is not clear.

3) The development of diagnostic tests: as of now the definitive evidence is only by real time PCR which needs sophisticated set up [39-41]. Considering the alarming figures ahead, point of care tests need to be developed. Also, since the virus is closely related to dengue virus, it may be useful to have an NS1 antigen based assay for early diagnosis which could be possibly be done in a routine laboratory or could be performed as a point of care test in the form of rapid card test $[42,43]$. Studies are required to develop and evaluate the serological markers of acute Zika infection as cross-reactive antibodies may yield false positive results.

4) Vaccine development: The National Institute for Allergy and Infectious Diseases (NIAID) is currently pursuing at least two approaches for a Zika vaccine [44]. First, a DNA based vaccine, and the other a live vaccine is being tried based on highly immunogenic approaches similar to those used for the closely related dengue virus. It might be possible to just swap Zika DNA, or a whole Zika virus, into the experimental vaccines since it is so similar. Bharat Biotech in India has become the first company to file patent for Zika vaccine, which will be tested in animal models and could provide affordable vaccine as has been done earlier by developing indigenous oral Rotavirus Vaccine (Rotavac) $[45,46]$. In a recent preclinical study, ZIKV purified inactivated virus vaccine candidate was found to give robust protection against the virus in rhesus monkeys; the vaccine may soon advance to human trials [47].

5) Vector control: Millions of people die/suffer every year from the mosquito-borne diseases such as malaria, dengue, chikungunya, and yellow fever. Now the danger of Zika virus is hovering around creating a global alert. Currently, various methods are being used for the vector (mosquito) control strategies, and these include chemical control (indoor residual spray, chemical larvicides, aerosol space spray, malathion fogging during outbreaks), biological control (use of larvivorous fish in ornamental tanks, fountains, use of biocides), personal prophylactic measures (use of mosquito repellent creams, liquids, coils, mats, screening of houses with wire mesh, insecticide treated bednets, and wearing of clothes that cover maximum surface area of body), community participation for detection of mosquito breeding sites for its elimination, environmental management and source reduction methods (filling of the breeding places, proper covering of stored water) $[23,48]$. All these methods have been implicated for the past many years; however, every year millions of cases of malaria, dengue, chikungunya, 
and yellow fever are reported from different parts of the world. The scientific community is also testing the novel approaches of vector control such as use of Genetically Modified (GM) males of Aedes mosquitoes [49]. Such mosquitoes carry a gene that hinders the development of their progeny and the second generation dies before they can reproduce. Field trials in Cayman Island and Brazil reported a reduction of mosquitoes up to $96 \%$ and $92 \%$ respectively [49]. However, it requires a large number of mosquitoes to be released into small area and eliminating one particular species of mosquitoes may have deleterious consequences in near future as it may lead to disturbance in food chain. Mosquitoes, and their larvae are an important source of food for birds, bats, fishes and frogs. This may also lead to emergence of some new vector, which may have the strong potential of transmitting even more deadly diseases [49]. Thus, every action needs close monitoring to prevent any adverse consequences.

6) Molecular characterisation of the virus: to identify any mutations that has facilitated the spread. In the past mutation in the envelope protein region (E1-A226V) of chikungunya virus led to adaptation to $A$. albopictus mosquitoes that caused an epidemic in a region lacking the typical vector [50]. The next generation whole genome sequencing could provide answers to such queries [51].

Recognizing that human health, animal health and environment health are linked inseparably, One Health concept has been launched to promote, improve and defend the health and well being of all the species by enhancing cooperation and collaboration between physicians, veterinarians, other scientific and health and environment professionals and by promoting management to achieve these goals $[52,53]$.

Due to recent Ebola outbreak in West Africa, various health agencies in the form of rapid response teams are in place to deal with the management, and control of outbreaks [54,55]. In India, due to recent viral outbreaks, rapid response teams along with staff of Integrated Disease Surveillance Programme (IDSP) are active [56], which could speed up the process of managing the Zika outbreak. Still, we require rapid action research teams that can focus on different biological aspects of Zika viral infection related to molecular epidemiology, pathogenesis, development of antivirals and vaccines. Therefore, we all must join our hands to cross the zig zag path of Zika virus infection.

\section{Conflict of Interest: None}

\section{References}

1. World Health Organization, Zika virus disease. World Health Organization, Geneva, Switzerland.

2. Zika virus, World Health Organisation declares Zika virus public health emergency. Guardian News and Media Limited, London, UK.

3. Is El nino helping spread Zika? Cable News Network, Atlanta, Georgia, USA.

4. Zika virus: Up to four million Zika cases predicted. BBC, London, UK.

5. Athletes told to stay away from water and placed under house arrest by coaches due to concerns over Zika. Daily Mail Online, London, UK.

6. World Health Organization, The history of Zika virus. World Health Organization, Geneva, Switzerland.

7. Smithburn KC (1952) Neutralizing antibodies against certain recently isolated viruses in the sera of human beings residing in East Africa. J Immunol 69: 223-234.
8. Kindhauser MK, Allen T, Frank V, Santhana R, Dye C (2016) Zika: the origin and spread of a mosquito-borne virus. Bulletin of the World Health Organization, World Health Organization, Geneva, Switzerland.

9. Cauchemez S, Besnard M, Bompard P, Dub T, Guillemette-Artur P, et al. (2016) Association between Zika virus and microcephaly in French Polynesia, 2013-2015: A retrospective study. Lancet 387: 2125-2132.

10. Butler D (2016) Zika virus: Brazil's surge in small-headed babies questioned by report. Nature 530: 13-14.

11. Microcephaly: What is the disease caused by Zika which damages babies' brains? Independent, London, UK.

12. Centers for Disease Control and Prevention, Zika and Guillain-Barré Syndrome. Centers for Disease Control and Prevention, Atlanta, Georgia, USA

13. World Health Organization, Countries and territories showing historical distribution of Zika virus, 1947 - 2016. World Health Organization, Geneva, Switzerland.

14. What will life be like for Brazil's generation of Zika babies? New Scientist, UK.

15. India fertile ground for Zika epidemic. The Financial Express, Noida, India.

16. Bogoch, II, Brady OJ, Kraemer MU, German M, Creatore MI, et al. (2016) Potential for Zika virus introduction and transmission in resource-limited countries in Africa and the Asia-Pacific region: a modelling study. The Lancet Infectious diseases 16: 1237-1245.

17. Why India should be concerned about the Zika virus? Daily News \& Analysis, Mumbai, India.

18. Rodriguez-Barraquer I, Solomon SS, Kuganantham P, Srikrishnan AK, Vasudevan CK, et al. (2015) The Hidden Burden of Dengue and Chikungunya in Chennai, India. PLoS Negl Trop Dis 9: 0003906.

19. Why Asia should worry about Zika too? BBC, London, UK.

20. Musso D, Gubler DJ (2016) Zika Virus. Clinical microbiology reviews 29: 487524.

21. Dejnirattisai W, Supasa P, Wongwiwat W, Rouvinski A, Barba-Spaeth G, et al. (2016) Dengue virus sero-cross-reactivity drives antibody-dependent enhancement of infection with zika virus. Nat Immunol 17: 1102-1108.

22. Paul LM, Carlin ER, Jenkins MM, Tan AL, Barcellona CM, Nicholson CO, Dengue Virus Antibodies Enhance Zika Virus Infection. bioRxiv, Cold Spring Harbor Laboratory, New York, USA.

23. National Guidelines for Zika Virus disease. Ministry of Health and Family Welfare. Government of India, India.

24. Number of Pregnant Women Received 3 ANC Check-ups in India. Government of India, India.

25. Centers for Disease Control and Prevention, Zika and Sexual Transmission. Centers for Disease Control and Prevention, Atlanta, Georgia, USA.

26. Centers for Disease Control and Prevention, Zika and Blood Transfusion. Centers for Disease Control and Prevention, Atlanta, Georgia, USA.

27. Howard CR, Fletcher NF (2012) Emerging virus diseases: can we ever expect the unexpected? Emerging microbes \& infections 1: 46.

28. Lerner H, Berg C (2015) The concept of health in One Health and some practical implications for research and education: what is One Health? Infect Ecol Epidemiol 5: 25300.

29. Gratz NG, Steffen R, Cocksedge W (2000) Why aircraft disinsection? Bull World Health Organ 78: 995-1004.

30. Russell RC, Paton R (1989) In-flight disinsection as an efficacious procedure for preventing international transport of insects of public health importance. Bull World Health Organ 67: 543-547.

31. Chahar HS, Bharaj P, Dar L, Guleria R, Kabra SK, et al. (2009) Co-infections with chikungunya virus and dengue virus in Delhi, India. Emerg Infect Dis 15: 1077-1080. 
32. Leroy EM, Nkoghe D, Ollomo B, Nze-Nkogue C, Becquart P, et al. (2009) Concurrent chikungunya and dengue virus infections during simultaneous outbreaks, Gabon, 2007. Emerg Infect Dis 15: 591-593.

33. Rohani A, Potiwat R, Zamree I, Lee HL (2009) Refractoriness of Aedes aegypti (Linnaeus) to dual infection with dengue and chikungunya virus. Southeast Asian J Trop Med Public Health 40: 443-448.

34. Dupont-Rouzeyrol M, O'Connor O, Calvez E, Daurès M, John M, et al. (2015) Co-infection with Zika and dengue viruses in 2 patients, New Caledonia 2014. Emerg Infect Dis 21: 381-382.

35. Dowall SD, Graham VA, Rayner E, Atkinson B, Hall G, et al. (2016) A susceptible mouse model for Zika virus infection. PLoS Negl Trop Dis 10: 4658.

36. Lazear HM, Govero J, Smith AM, Platt DJ, Fernandez E, et al. (2016) A Mouse Model of Zika Virus Pathogenesis. Cell Host Microbe 19: 720-730.

37. Rossi SL, Tesh RB, Azar SR, Muruato AE, Hanley KA, et al. (2016) Characterization of a Novel Murine Model to Study Zika Virus. Am J Trop Med Hyg 94: 1362-1369.

38. Tang H, Hammack C, Ogden SC, Wen Z, Qian X, et al. (2016) Zika Virus Infects Human Cortical Neural Progenitors and Attenuates Their Growth. Cell stem cell 18: 587-590.

39. Faye O, Faye O, Diallo D, Diallo M, Weidmann M, et al. (2013) Quantitative real-time PCR detection of Zika virus and evaluation with field-caught mosquitoes. Virol j 10: 311.

40. Xu MY, Liu SQ, Deng CL, Zhang QY, Zhang B (2016) Detection of Zika virus by SYBR green one-step real-time RT-PCR. J Virol methods 236: 93-97.

41. Faye O, Faye O, Dupressoir A, Weidmann M, Ndiaye M, et al. (2008) Onestep RT-PCR for detection of Zika virus. J Clin Virol 43: 96-101.

42. Singh MP, Goyal K, Ratho RK (2010) Nonstructural protein NS1: giving a new structure to dengue diagnosis. J Clin Microbiol 48: 4688-4689.

43. Singh MP, Majumdar M, Singh G, Goyal K, Preet K, et al. (2010) NS1 antigen as an early diagnostic marker in dengue: report from India. Diagn Microbiol Infect Dis 68: 50-54.
44. Could We Have a Zika Vaccine Soon? NBC News, New York, USA.

45. Bharat Biotech gets breakthrough in developing Zika vaccine. The Economic Times, Uttar Pradesh, India.

46. PM Narendra Modi Launches Rotavirus Vaccine Developed in India. NDTV, New Delhi, India

47. American Association for the Advancement of Science, Zika Vaccines Offer Full Protection to Monkeys, Science Reports. American Association for the Advancement of Science, Washington, D.C., USA.

48. Vector control measures. National Vector Borne Disease Control Programme, Government of India, New Delhi, India.

49. Would it be wrong to eradicate mosquitoes? BBC, London, UK.

50. Tsetsarkin KA, Vanlandingham DL, McGee CE, Higgs S (2007) A single mutation in chikungunya virus affects vector specificity and epidemic potential. PLoS Pathog 3: 201.

51. First complete genome sequence of the Zika virus released. ScienceDaily, Rockville, Maryland, USA.

52. Rabinowitz PM, Kock R, Kachani M, Kunkel R, Thomas J, et al. (2013) Toward proof of concept of a one health approach to disease prediction and control. Emerging infectious diseases 19

53. Kakkar M, Abbas SS, Roadmap to Combat Zoonoses in India (RCZI) initiative (2011) One health: moving from concept to reality. Lancet Infect Dis 11: 808.

54. McCarthy M (2014) US deploys rapid response teams to hospitals with Ebola cases. BMJ 349: 6266.

55. Sprenger M, Coulombier D (2014) Preparedness is crucial for safe care of Ebola patients and to prevent onward transmission in Europe - outbreak control measures are needed at its roots in West Africa. Euro Surveill 19: 20925

56. India prepares for Zika virus, health ministry readies testing kits. Hindustan Times, New Delhi, India. 